\title{
Conformational Switching on Platinum(II) Coordination Plane Triggered by Oxalate Anion
}

\author{
Ki-Hyun Kim, Rira Ju, Hae-Jo Kim, ${ }^{\dagger}$ Moo-Jin Jun, ${ }^{\dagger}$ and Kwan Mook Kim* \\ Department of Nano-Chemistry, Ewha Womans University, Seoul 120-750, Korea. E-mail: kkmook@ewha.ac.kr \\ ${ }^{\dagger}$ Department of Chemistry, Hankuk University of Foreign Studies, Yongin 449-791, Korea \\ *KISTI ReSEAT program senior fellow, Korea Institute of Science and Technology Information, Seoul 130-742, Korea \\ Received June 29, 2011, Accepted July 21, 2011
}

Key Words : Conformational switching, Oxalate sensor, Diamine-platinum(II) complex, Host-guest chemistry

A molecular conformational switching has been of growing interest in association with molecular electronics, ${ }^{1}$ machines $^{2}$ and sensors. ${ }^{3}$ External stimuli such as a transition metal, ${ }^{4}$ light, ${ }^{5}$ a redox reaction, ${ }^{6} \mathrm{pH}_{\text {variation }}{ }^{7}$ and guest molecules ${ }^{8}$ were used to control the thermodynamic equilibrium between the conformers. For example, complexation with lewis acid, $\mathrm{TiCl}_{4}$, changes the conformation of arenedicarboxamide from syn to anti atropisomer. ${ }^{4}$ The specially designed ligand is programmed to have syn conformer by the application of the transition metal complex $\mathrm{PdCl}_{2}(\mathrm{PhCN})_{2}{ }^{9}$

$N$-Alkyl-substituted-ethylenediamine complexes of platinum(II) are well known to adopt syn and anti conformers which are inter-converted fast in aqueous solutions. ${ }^{10}$ We have been interested in developing a conformational switching system by exploiting this behavior, thus designed complex 1 with two uryl groups which are well known to bind carboxylates. ${ }^{11}$ We speculated that the thermodynamic ratio of syn to anti conformer of 1 would be influenced by a suitable dicarboxylate anion such as oxalate which is a biologically important anion. ${ }^{12}$

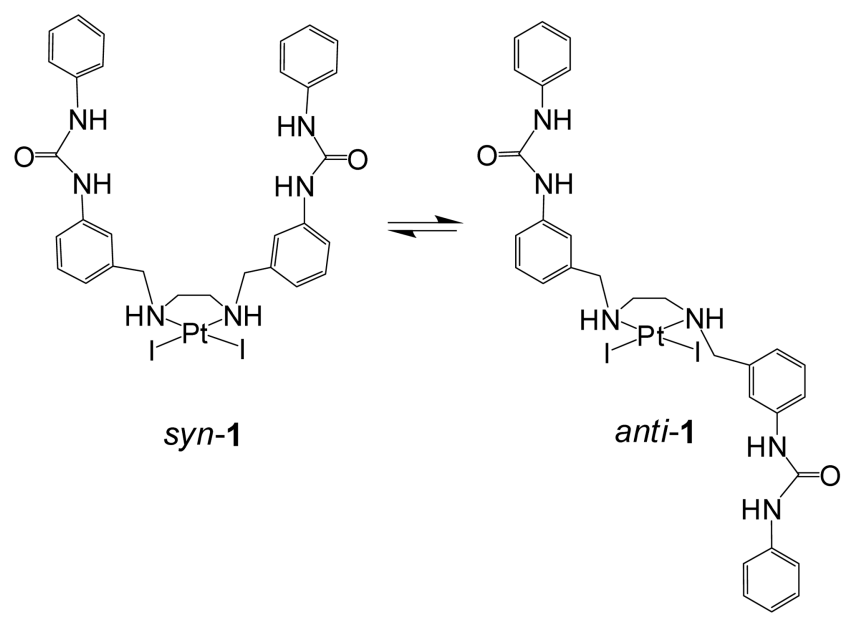

Ligand $N, N$ '-bis-(3-phenyluryl-benzyl)-ethylenediamine (L), was synthesized by the reductive amination of 3phenyluryl-benzaldehyde by ethylenedamine and $\mathrm{NaBH}_{4}$ (Scheme 1). The compound, 3-phenyluryl-benzaldehyde, was prepared by the PCC oxidation of 3-phenylurylbenzylalcohol. Platinum complex 1 was obtained as a crude

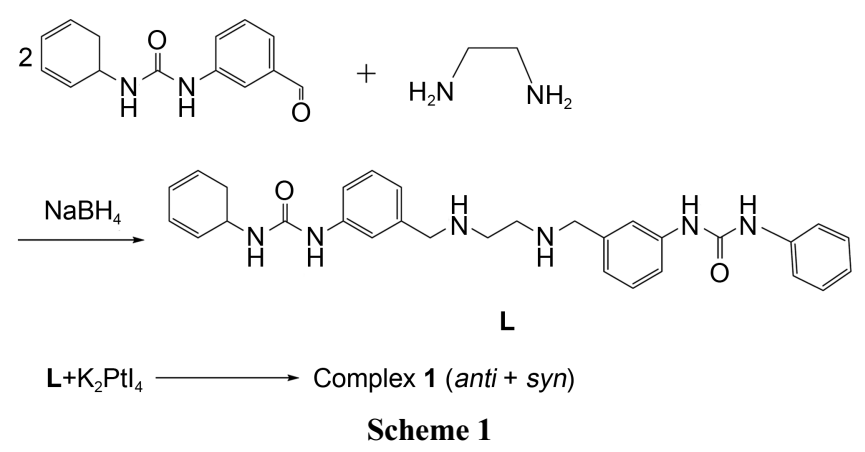

precipitate by the treatment of $\mathbf{L}$ with $\mathrm{K}_{2} \mathrm{PtI}_{4}$ in water/ methanol cosolvent. The platinum complex was further purified by recrystallization in DMF/water.

Complex $\mathbf{1}$ is insoluble in water, only marginally soluble in acetone, acetonitrile and methanol, freely soluble in DMF, and decomposes in DMSO probably due to the coordination of the solvent to the metal complex. The analysis of ${ }^{1} \mathrm{H}$ NMR spectrum for the crude product revealed that the syn conformer exists in less amount than the anti conformer with the initial syn/anti ratio of $0.47 / 1$ in DMF- $d_{7}$. We obtained similar results with the crude products from several experimental batches. At equilibrium, however, the syn/anti ratio of the crude complex changed to $1.55 / 1$ in DMF- $d_{7}$. The anti-syn conversion rate of $\mathbf{1}$ in DMF- $d_{7}$ was so slow that more than 14 days were required to reach the equilibrium, which was much slower compared to the known inversion rates ${ }^{10}$ of other platinum(II) complexes in aqueous solution.

Figure 1 shows time-dependent partial ${ }^{1} \mathrm{H}$ NMR spectra in the region of uryl $\mathrm{NH}$ and ethylene $\mathrm{CH}$ signals when 1.0 equivalent oxalic acid was added to 1 in the presence of excess base triethylamine in DMF- $d_{7}$. The concentration of 1 was kept to be $20 \mathrm{mM}$. The experiment started with the crude complex 1 which has more anti conformer than syn conformer. The two uryl NH signals of the syn conformer shift to more downfield region than those of the anti conformer with the addition of oxalate anions. The more downfield signals imply the stronger hydrogen bonding between the oxalate and the uryl group. The ethylene $\mathrm{CH}$ signals around $5.0 \mathrm{ppm}$ change to $5.1 \mathrm{ppm}$, which certainly reflect the conformational changes from anti to syn of 1 . The 

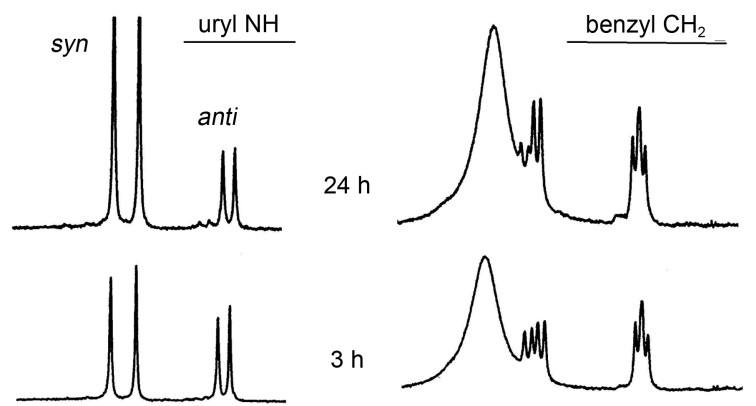

$3 \mathrm{~h}$
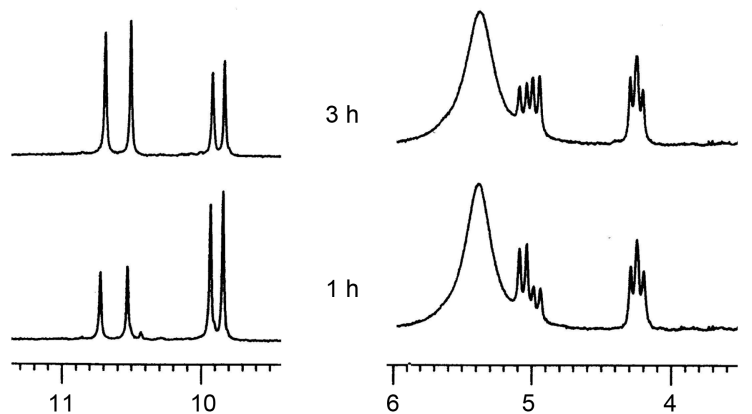

Figure 1. Time-dependent ${ }^{1} \mathrm{H}$ NMR spectra showing the conformational switching of $\mathbf{1}$ from anti-excess to syn-excess in the presence of 1 equiv. oxalate.

time dependent spectra in Figure 1 demonstrates that the syn/anti ratio increases notably with the addition of 1.0 equiv oxalate. At final equilibrium, the amount of syn conformer exceeds that of anti one by the ratio of $\sim 4: 1$. This equlibrium ratio in the presence of oxalate is significantly greater than the ratio of the solution without oxalate.

The same ${ }^{1} \mathrm{H}$ NMR studies demonstrated as in Figure 1 were applied to other dicarboxylates, malonate and succinate. Syn/anti ratios at final equilibrium were 2.22/1 and 2.13/1 for malonate and succinate, respectively. The syn/anti ratio $(4 / 1)$ of the oxalate solution at equilibrium is apparently larger than those of malonate and succinate.

Figure 2 shows the dependence of the chemical shifts of an NH proton peak in ${ }^{1} \mathrm{H}$ NMR on the change of the concentrations of oxalate, malonate and succinate. The concentration of the base TEA has been kept to be 4 equivalents of the diacids in this study, while the concentration of $\mathbf{1}$ was kept to be $20 \mathrm{mM}$. The chemical shift of NH uryl proton appears at $9.08 \mathrm{ppm}$ in the solution without any substrate,

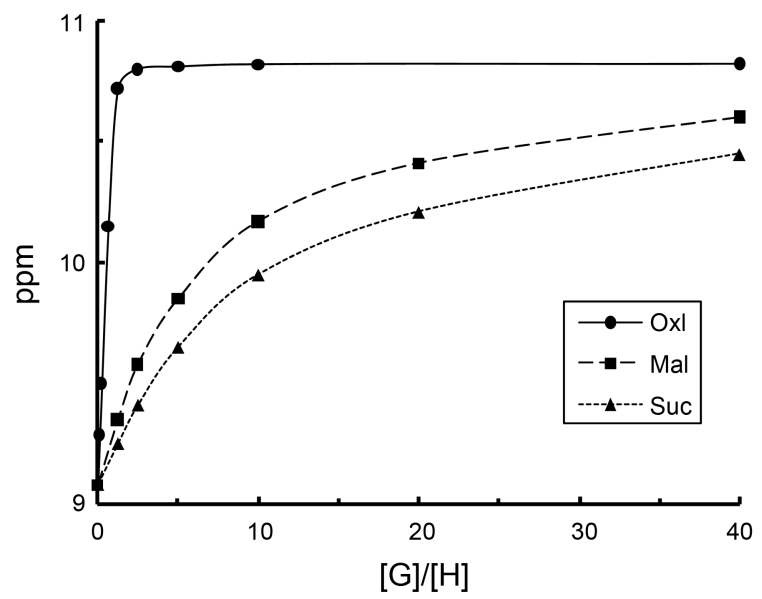

Figure 2. The dependence of ${ }^{1} \mathrm{H}$ NMR chemical shift of one NH upon the addition of dicarboxylate anions. $\bullet$ : oxalate, $\mathbf{\square}$ : malonate, $\boldsymbol{\Delta}$ : succinate.

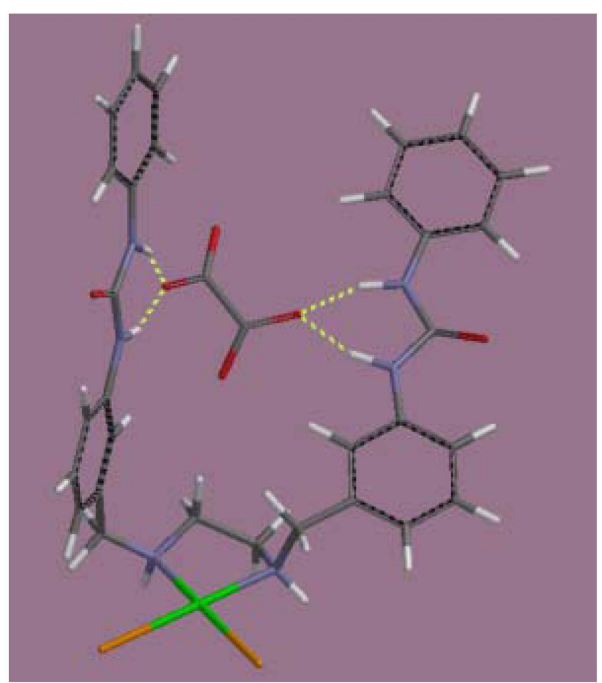

Figure 3. Predicted structure of syn-1 binding oxalate calculated by Molecular Mechanics.

and saturates to $10.81 \mathrm{ppm}$ with the addition of oxalate. Based on the 1:1 binding model between 1 and dicarboxylate, the dissociation constant of oxalate for the platinum complex, syn-1, was calculated to be $(3.95 \pm 0.20)$ $\times 10^{-4} \mathrm{M}$, whose binding affinity is much stronger than $(1.12$ $\pm 0.04) \times 10^{-1} \mathrm{M}$ of malonate and $(1.81 \pm 0.08) \times 10^{-1} \mathrm{M}$ of succinate. Oxalate exhibits a prominent syn selectivity of complex 1 with more than 280 -fold binding affinity over other structurally similar dicarboxylates.

The 1:1 binding of oxalate to syn-1 can be explicable by the energy minimum structure in Figure 3, which suggests the optimum interaction between oxalate and two uryl groups. ${ }^{13}$ Two uryl groups fix ideally the oxalate anion between them. This binding mode is considered to induce complex 1 to take the syn conformation for the optimal hydrogen bonds.

For comparison, we also prepared the ligand, $N, N^{\prime}$-bis-(4phenyluryl-benzyl)-ethylenediamine $\left(\mathbf{L}^{\prime}\right)$, which has the uryl group at para postion.

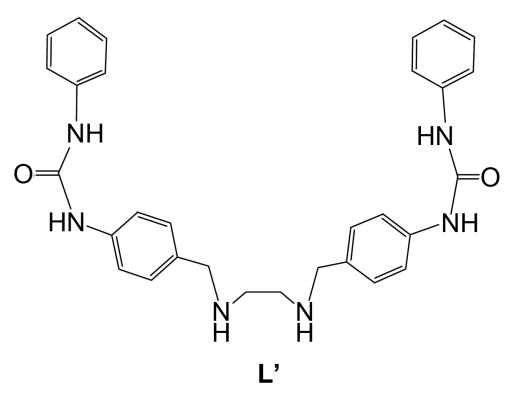

The platinium complex, $\left(\mathbf{L}^{\prime}\right) \mathrm{PtI}_{2}(\mathbf{2})$, was prepared by the same method as $\mathbf{1}$ using $\mathbf{L}^{\prime}$ and $\mathrm{K}_{2} \mathrm{PtI}_{4}$. The syn/anti ratio of the crude compound 2 was $0.5 / 1$ in DMF- $d$, and the ratio in the equilibrium was $1.5 / 1$, which are very similar to those of compound $\mathbf{1}$. However, the two uryl groups in complex $\mathbf{2}$ might be located in unfavorable positions, so that its binding affinity toward oxalate anion is expected to be less than that 
Table 1. The syn/anti ratios of $\mathbf{1}$ and $\mathbf{2}$ at different conditions in DMF- $d_{7}$

\begin{tabular}{lc}
\hline \multicolumn{1}{c}{ Solutions } & syn/anti ratio \\
\hline $\mathbf{1}$ in initial & $0.47 / 1$ \\
$\mathbf{1}$ at equilibrium & $1.55 / 1$ \\
$\mathbf{1}+4$ TEA +1.0 Oxal & $4.00 / 1$ \\
$\mathbf{1}+4$ TEA $+1.0 \mathrm{Mal}$ & $2.22 / 1$ \\
$\mathbf{1}+4$ TEA +1.0 Succ & $2.13 / 1$ \\
$\mathbf{2}$ in initial & $0.5 / 1$ \\
$\mathbf{2}$ at equilibrium & $1.5 / 1$ \\
$\mathbf{2}+4$ TEA +1.0 Oxal & $1.6 / 1$ \\
\hline
\end{tabular}

of $\mathbf{1}$. Indeed, the syn/anti ratio of compound $\mathbf{2}$ in the presence of oxalate was $\sim 1.6 / 1$, which is almost same with that in the absence of oxalate. The comparison of complexes 1 and $\mathbf{2}$ demonstrates certainly that the binding mode in Figure 3 is a key factor driving $\mathbf{1}$ into the syn conformation.

Table 1 summarizes the syn/anti ratios obtained in this work in different conditions.

In conclusion, we have developed molecular switching system triggered by oxalate anion operating on platinum square planar coordination plane by controlling well-known alkyl inversion process on nitrogens. This work shows that oxalate anion disturbs the thermodynamic equilibrium between syn and aniti conformer and induces the syn conformer to be produced in excess. Thus oxalate may be regarded as a trigger that bring about the conformational change of complex 1. This behavior might be exploited to develop sensors for the kidney stone disease-related oxalate anions. ${ }^{3}$

\section{Experimental Section}

$\left(N, N^{\prime}\right)$-Bis(3-phenyluryl-benzyl)-ethylenediamine (L). A mixture of 3-phenyluryl-benzaldedyde $(2.0 \mathrm{~g}, 8.3 \mathrm{mmol})$ and ethylenediamine $(0.25 \mathrm{~g}, 4.1 \mathrm{mmol})$ in ethanol $(30 \mathrm{ml})$ was refluxed for $18 \mathrm{~h}$. The solvent was then evaporated. The residue was reacted with $\mathrm{NaBH}_{4}(0.38 \mathrm{~g}, 10.0 \mathrm{mmol})$ in THF $(50 \mathrm{~mL})$ at room temperature for $4 \mathrm{~h}$. The solvent was evaporated and the product was extracted with chloroform. The crude product was purified by recrystallization in $\mathrm{CHCl}_{4}$ and hexane to obtain $1.8 \mathrm{~g}$ of $\mathbf{L}$ (yield: $83 \%$ ). ${ }^{1} \mathrm{H}$ NMR (250 MHz, DMSO- $\left.d_{6}\right): \delta$ (TMS) 6.8-7.5 (m, 18H, aromatic), 3.75 (s, 4H, benzyl), 2.73 (s, 4H, ethylene). HRMS (FAB) calcd for $\mathrm{C}_{30} \mathrm{H}_{32} \mathrm{~N}_{6} \mathrm{O}_{2}$ : 508.6285; found: 508.6208.

$\left(N, N^{\prime}\right)$-Bis(4-phenyluryl-benzyl)-ethylenediamine $\left(\mathrm{L}^{\prime}\right)$. The compound $\mathbf{L}^{\prime}$ was obtained by the same procedures as $\mathbf{L}$ starting from 4-phenyluryl-benzaldedyde and ethylenediamine. ${ }^{1} \mathrm{H}$ NMR (250 MHz, DMSO- $d_{6}$ ): $\delta$ (TMS) 6.8-7.5 (m, 18H, aromatic), 3.56 (s, 4H, benzyl), 2.52 (s, 4H, ethylene). HRMS (FAB) calcd for $\mathrm{C}_{30} \mathrm{H}_{32} \mathrm{~N}_{6} \mathrm{O}_{2}: 508.6285$; found: 508.6252 .

(L)PtI $\mathbf{2}$ (1). $\mathrm{K}_{2} \mathrm{PtCl}_{4}(1.0 \mathrm{~g}, 2.5 \mathrm{mmol})$ and $\mathrm{KI}(7.0 \mathrm{~g}, 42$ mmol) was stirred in water $(20 \mathrm{~mL})$ for $1 \mathrm{~h}$. $\mathbf{L}(1.4 \mathrm{~g}, 2.7$ mmol) in $20 \mathrm{~mL}$ acetone was added dropwise to the solution, and the resulting solution was refluxed for $24 \mathrm{~h}$. Fine yellow precipitate, $(\mathbf{L}) \mathrm{PtI}_{2}(\mathbf{1})$, was formed in the reaction solution, which was separated by filtration and washed with ethanol, diethylether and dried under vacuum. The product was a mixture of anti-1 and $s y n-1$ with total yield of $85 \%$. ${ }^{1} \mathrm{H}$ NMR $\left(250 \mathrm{MHz}, \mathrm{DMF}-d_{7}\right)$ for anti-1: $\delta$ (TMS) $9.84(\mathrm{~s}, 2 \mathrm{H}$, uryl NH), $9.76(\mathrm{~s}, 2 \mathrm{H}$, uryl NH), 8.03 (s, $2 \mathrm{H}$, aromatic), 6.9-7.8 (m, 16H, aromatic), $4.78(\mathrm{~d}, 2 \mathrm{H}$, benzyl), 3.99 (d, 2H, benzyl), 3.05 (dd, 2H, ethylene), 2.41 (dd, 2H, ethylene). ${ }^{1} \mathrm{H}$ NMR (250 MHz, DMF- $d_{7}$ ) for $s y n-1$ : $\delta$ (TMS) 9.85 (s, 2H, uryl NH), 9.77 (s, 2H, uryl NH), 8.03 (s, 2H, aromatic), 6.9-7.8 (m, 16H, aromatic), $4.90(\mathrm{~d}, 2 \mathrm{H}$, benzyl), 4.02 (d, 2H, benzyl), 2.72 (dd, 2H, ethylene), 2.61 (dd, $2 \mathrm{H}$, ethylene).

(L') $\mathbf{P t I}_{2}$ (2). This was obtained by the same procedures as 1 starting with $\mathbf{L}^{\prime}$. The ${ }^{1} \mathrm{H}$ NMR was same with $\mathbf{1}$ except in the aromatic region.

Determination of Binding Constant by NMR Titration Method. ${ }^{14}$ Our analysis is based on a simple 1:1 binding equilibrium model. A host $(\mathbf{H})$ and a guest $(\mathbf{G})$ are assumed be in equilibrium with the complex (HG). The observed chemical shift $\left(d_{\text {obs }}\right)$ of a selected proton on the host is a weighted average of that proton's chemical shift in two possible environments - the chemical shifts in the unbound host environment $\left(\delta_{\mathrm{H}}\right)$ and the chemical shift in the bound host environment $\left(\delta_{\mathrm{HG}}\right)$. If the initial host concentration is defined to be $[H]_{\mathrm{o}}$, then Equation (1) applies.

$$
\delta_{\mathrm{obs}}=\frac{[\mathrm{H}]_{\mathrm{o}}-[\mathrm{HG}]}{[\mathrm{H}]_{\mathrm{o}}} \delta_{\mathrm{H}}+\frac{[\mathrm{HG}]}{[\mathrm{H}]_{\mathrm{o}}} \delta_{\mathrm{HG}}
$$

Defining $\Delta \delta_{\max }$ to be the difference in chemical shift for the two environments between complex and free host, the observed chemical shift can be expressed as a function of $[\mathrm{H}]_{\mathrm{o}},[\mathrm{G}]_{\mathrm{o}}, \mathrm{K}_{\mathrm{d}}$ and $\delta_{\mathrm{H}}$.

$$
\delta_{\mathrm{obs}}=\delta_{\mathrm{H}} \frac{\left([\mathrm{H}]_{\mathrm{o}}+[\mathrm{G}]_{\mathrm{o}}+\mathrm{K}_{\mathrm{d}}\right)-\sqrt{\left([\mathrm{H}]_{\mathrm{o}}+[\mathrm{G}]_{\mathrm{o}}+\mathrm{K}_{\mathrm{d}}\right)^{2}-4[\mathrm{H}]_{\mathrm{o}}[\mathrm{G}]_{\mathrm{o}}}}{2[\mathrm{H}]_{\mathrm{o}}} \Delta \delta_{\max }
$$

Equation (2) was used for the binding affinity determination by applying a linear regression method implemented in the program Sigma plot.

Acknowledgments. This work was supported by National Research Foundation of Korea Grant funded by the Korean Government (SRC Program: 2011-0001334) and by RPGrant 2010 of Ewha Womans University. One of the authors, M.-J. Jun, is grateful to the KISTI ReSEAT program for the support.

\section{References}

1. (a) Feringa, B. L.; van Delden, R. A.; ter Wiel, M. K. J. Pure Appl. Chem. 2003, 75, 563-575. (b) Seminario, J. M.; Derosa, P. A.; Bastos, J. L. J. Am. Chem. Soc. 2002, 124, 10266-10267.

2. Balzani, V.; Credi, A.; Raymo, F. M.; Stoddart, J. F. Angew. Chem. Int. Ed. 2000, 39, 3348-3391.

3. Thomas, S. W., III; Joly, G. D.; Swager, T. M. Chem. Rev. 2007, 107, 1339-1386.

4. Clayden, J.; Vallverdú, L.; Clayton, J.; Helliwell, M. Chem. Commun. 2008, 561-563. 
5. Sharma, A.; Reva, I.; Fausto, R. J. Am. Chem. Soc. 2009, 131, 8752-8753.

6. Zahn, S.; Canary, J. W. J. Am. Chem. Soc. 2002, 124, 9204-9211

7. Yamasaki, R.; Tanatani, A.; Azumaya, I.; Saito, S.; Yamaguchi, K.; Kagechika, H. Org. Lett. 2003, 5, 1265-1267.

8. Katoono, R.; Kawai, H.; Fujiwara, K.; Suzuki, T. Tetrahedron Lett. 2006, 47, 1513-1518.

9. Chong, Y. S.; Smith, M. D.; Shimizu, K. D. J. Am. Chem. Soc. 2001, 123, 7463-7464

10. (a) Haake, P.; Turley, P. C. J. Am. Chem. Soc. 1968, 90, 22932295. (b) Erickson, L. E. J. Am. Chem. Soc. 1969, 91, 6284-6290.
11. Park, H.; Kim, K. M.; Lee, A.; Ham, S.; Nam, W.; Chin, J. J. Am. Chem. Soc. 2007, 129, 1518-1519.

12. (a) Robertson, W. G.; Hughes, H. Scanning Microsc. 1993, 7, 391. (b) Tang, L.; Park, J.; Kim, H.-J.; Kim, Y.; Kim, S. J.; Chin, J.; Kim, K. M. J. Am. Chem. Soc. 2008, 130, 12606.

13. The optimization has been carried out by Molecular Mechanics with the program Spartan '04 of Wavefunction Inc.

14. Wilcox, C. S. Frontiers in Supramolecular Organic Chemistry and Photochemistry; Schneider, H.-J., Durr, H., Eds.; VCH: Weinheim, 1991; 123-143. 